\title{
Direct Laser Desorption Ionization of Endogenous and Exogenous Compounds from Insect Cuticles: Practical and Methodologic Aspects
}

\author{
Joanne Y. Yew, ${ }^{1,2,3}$ Jens Soltwisch, ${ }^{1}$ Alexander Pirkl, ${ }^{1}$ Klaus Dreisewerd ${ }^{1}$ \\ ${ }^{1}$ Institute of Medical Physics and Biophysics, Robert-Koch-Str. 31, 48149 Münster, Germany \\ ${ }^{2}$ Temasek Life Sciences Laboratory, Singapore \\ ${ }^{3}$ Department of Biological Sciences, National University of Singapore, Singapore
}

\begin{abstract}
We recently demonstrated that ultraviolet laser desorption ionization orthogonal time-of-flight mass spectrometry (UV-LDI o-TOF MS) could be used for the matrix-free analysis of cuticular lipids (unsaturated aliphatic and oxygen-containing hydrocarbons and triacylglycerides) directly from individual Drosophila melanogaster fruit flies (Yew, J. Y.; Dreisewerd, K.; Luftmann, H.; Pohlentz, G.; Kravitz, E. A., Curr. Biol. 2009, 19, 1245-1254). In this report, we show that the cuticular hydrocarbon, fatty acid, and triglyceride profiles of other insects and spiders can also be directly analyzed from intact body parts. Mandibular pheromones from the jaw of a queen honey bee are provided as one example. In addition, we describe analytical features and examine mechanisms underlying the methodology. Molecular ions of lipids can be generated by direct UV-LDI when non-endogenous compounds are applied to insect wings or other body parts. Current sensitivity limits are in the $10 \mathrm{pmol}$ range. We show also the dependence of ion signal intensity on collisional cooling gas pressure in the ion source, laser wavelength (varied between $280-380 \mathrm{~nm}$ and set to $2.94 \mu \mathrm{m}$ for infrared LDI), and laser pulse energy.
\end{abstract}

Key words: UV-LDI MS, FALDI, Cuticular hydrocarbons, Triglycerides, Drosophila melanogaster, Arthropods, Pheromones

\section{Introduction}

$\mathrm{T}$ he standard method for the analysis of hydrocarbons (HCs) is gas chromatography coupled with mass spectrometry (GC/MS). This method has been used extensively to characterize lipids expressed on the cuticular surface of insects [1]. Some of these exogenous lipids serve as communication cues (pheromones) and can greatly

Electronic supplementary material The online version of this article (doi:10.1007/s13361-011-0110-3) contains supplementary material, which is available to authorized users.

Correspondence to: Klaus Dreisewerd; e-mail: dreisew@uni-muenster.de influence behaviors such as mate choice and aggregation [1]. In addition, pheromones have been manipulated in order to control the population growth of agricultural pests and disease-bearing insects [2]. Recently, we showed that ultraviolet laser desorption ionization orthogonal time-of-flight mass spectrometry (UV-LDI o-TOF MS) allows analysis of cuticular hydrocarbons and triglycerides directly from the external cuticle of individual fruit flies [3-5]. Direct UVLDI MS provides spatially-resolved analysis of HCs without chemical extraction or the addition of a matrix to the cuticle. Moreover, low volatility, higher molecular weight polar compounds [e.g., long-chain aliphatic HCs, oxygen-containing $\mathrm{HCs}$, fatty acids (FA), and triacylglycerides (TAGs)] are amenable to analysis. Using this method, several new 
oxygen-containing hydrocarbons and a novel sex pheromone were characterized from D. melanogaster [3]. One disadvantage of the LDI MS method is that saturated aliphatic HCs (alkanes) are not detectable.

In this article, we show that chemical profiles of cuticles from other species of insects and spiders can also be analyzed by UV-LDI mass spectrometry. Furthermore, we demonstrate that lipids are detected with picomole sensitivity when exogenously applied to insect wings. Thus, the cuticle itself can be utilized as a substrate to facilitate UV-LDI MS analysis. To better characterize the mechanisms contributing to the phenomenon of flyassisted laser desorption/ionization (FALDI), we investigated the influence of the laser wavelength, laser fluence, and buffer gas pressure in the ion source of the o-TOF mass spectrometer.

\section{Experimental}

\section{Chemicals}

Z-11-hexadecen-1-ol $\left(\mathrm{C}_{16} \mathrm{H}_{32} \mathrm{O}\right)$, Z-9-hexadecenal $\left(\mathrm{C}_{16} \mathrm{H}_{30} \mathrm{O}\right)$, and Z-9-hexadecen-1-yl acetate $\left(\mathrm{C}_{18} \mathrm{H}_{34} \mathrm{O}_{2}\right)$ were from ISCA Technologies (Riverside, CA, USA). All other commercial chemicals were from Sigma-Aldrich (München, Germany). Crude extracts of Drosophila melanogaster cuticle were prepared by placing 20 flies of both sexes in $200 \mu \mathrm{L}$ of hexane or chloroform/methanol (2:1) for $10 \mathrm{~min}$ at room temperature. The supernatant was removed, evaporated using a gentle stream of $\mathrm{N}_{2}$, and stored at $-20^{\circ} \mathrm{C}$. Samples were reconstituted in $20 \mu \mathrm{L}$ of hexane or chloroform/ methanol (2:1) prior to analysis.

\section{Animals}

Drosophila melanogaster (Canton S) husbandry was performed as described [3]. Bees were kind gifts from local bee keepers. Other arthropods were serendipitous findings; these animals were not classified with respect to sex and species.

\section{Sample Preparation}

Whole insects or parts of the animals were prepared on glass coverslips using adhesive (G304; Plano, Wetzlar, Germany). The coverslips were mounted into a milled-out $(1.8 \mathrm{~mm}$ depth), custom-made sample plate holder using $200 \mu \mathrm{m}$ thick sticky pads. FALDI MS analyses were performed with wings from $D$. melanogaster bees or wasps. To reduce the chemical background from endogenous compounds, fly wings were partially washed by soaking in hexane for $10 \mathrm{~min}$. Analyte solutions, dissolved in hexane, were applied onto wings using pipettes. Standard matrix-assisted laser desorption ionization (MALDI) sample preparations were produced by mixing 2,5-dihydoxybenzoic acid (DHB) matrix $(10 \mathrm{mg} / \mathrm{mL})$ and analyte solutions on glass coverslips followed by air-drying.

\section{UV/IR-MALDI-o-TOF Mass Spectrometer}

The o-TOF mass spectrometer has been described in detail previously [6]. This instrument is a custom-made hybrid mass spectrometer that consists of an oMALDI 2 ion source, as used in QSTAR mass spectrometers (AB SCIEX, Concord, CA, USA), and a prOTOF-like (Perkin Elmer, Waltham MA, USA) quadrupole transfer-mass analyzer unit. A few measurements were also made with a commercial MALDI QSTAR instrument (pulsar-I, AB SCIEX; see below). In addition to the standard $\mathrm{N}_{2}$ UV-laser (337NDSx; Spectra Physics, Mountain View, CA, USA; $\lambda=$ $337 \mathrm{~nm} ; \tau_{\text {pulse }} \sim 3 \mathrm{~ns}$; maximum repetition rate, $30 \mathrm{~Hz}$ ), a Nd:YAG laser (Surelite II; Continuum, Darmstadt, Germany; $\lambda=532 \mathrm{~nm}$; $\tau_{\text {pulse }} \sim 6 \mathrm{~ns}$ ) pumped dye laser (FL 2001; Lambda Physik, Göttingen, Germany; repetition rate, $10 \mathrm{~Hz}$ ) and an infrared (IR) Er:YAG laser (Speser; Spektrum Laser $\mathrm{GmbH}$, Berlin, Germany; $\lambda=2.94 \mu \mathrm{m} ; \tau_{\text {pulse }} \sim 150 \mathrm{~ns}$; operated at $2 \mathrm{~Hz}$ ) are available on the o-TOF instrument for UV- and IR-LDI MS, respectively. Using a set of dyes and a BBO crystal for second harmonic generation, the dye laser can be tuned continuously between 280 and $380 \mathrm{~nm}$. In one experiment, a fundamental of the dye laser of $560 \mathrm{~nm}$ was employed for comparison. The emission wavelength was monitored using a StellarNet GREEN-Wave spectrometer (STE-GW-UV; Laser 2000, Wessling, Germany). The UV laser beams were delivered to the sample via an optical fiber system. A standard silica fiber with $200 \mu \mathrm{m}$ core diameter and $\sim 2 \mathrm{~m}$ length was used for delivery of the beam of the nitrogen laser [8]; for delivery of the dye laser beam, a second fiber with a core diameter of $200 \mu \mathrm{m}$ that was $10 \mathrm{~m}$ in length (SFS200/220y, NA 0.12; Fiberguide Industries, Stirling, NY, USA) was used. The fiber end surfaces were imaged 1:1 onto the sample using a custom-made telescope $\left(\mathrm{f}_{1,2}=15 \mathrm{~mm}, \phi_{\text {lenses }}=10 \mathrm{~mm}\right.$ ) producing an approximately "flat-top" intensity profile of about $200 \times 230 \mu \mathrm{m}^{2}$ in the focal plane [7]; the angle of incidence of the laser beam is $\sim 30^{\circ}$ relative to the ion optical axis. A custom-made optomechanical adjustment unit served for compensation of chromatic aberration (i.e., the wavelength-dependent focal lengths). Laser pulse energies were determined after the beam passed the glass fiber and the telescope unit using a commercial energy meter. After correction for reflection losses at the $\mathrm{CaF}_{2}$ vacuum window these values correspond to the laser energy supplied to the sample. A custom-made pyroelectric detector was furthermore used in combination with a beam splitter to monitor on-line the stability of the dye laser output.

The beam of the IR laser was focused onto the sample with a plano-convex infrasil lens to a spot of $\sim 200-300 \mu \mathrm{m}$ in diameter $\left(1 / \mathrm{e}^{2}\right.$-definition); the focal intensity profile of the IR laser beam was near-Gaussian; the angle of incidence relative to the ion optical axis was $\sim 30^{\circ}$. The 
three lasers exhibited pulse-to-pulse energy variations (standard deviations) of $\sim 2 \%\left(\mathrm{~N}_{2}\right.$ laser), $2 \%-10 \%$ (dye laser, depending on the emission wavelength), and $\sim 10 \%-$ 20\% (Er:YAG laser). Laser fluences were adjusted with dielectric attenuators (Er:YAG and dye laser) and a graduated neutral density filter $\left(\mathrm{N}_{2}\right.$ laser $)$. The ion extraction voltage applied between sample plate and extraction cone (the latter having a central aperture of $2 \mathrm{~mm}$ ) was set to $45 \mathrm{~V}$. Argon was used as buffer gas for collisional cooling and focusing $[6,8]$. Pressure in the ion source and in the interface region (that houses the collisional focusing transfer quadrupole) was controlled via two gas inlets. The pressure in the ion source was monitored with a capacitive pressure gauge (DVR 5; Vaccubrand, Wertheim, Germany) as described previously [6]. The pressure in the quadrupole ion guide was measured with a Pirani gauge. This pressure was kept between $1 \times 10^{-3}$ and a few $10^{-2}$ mbar. After passing the differentially-pumped interface region, ions were accelerated orthogonally using a TOF voltage of $10 \mathrm{kV}$. Typically, a pusher plate frequency of $7.5 \mathrm{kHz}$ and a bin width for the time-to-digital conversion of 132 ps were applied. Depending on the sample, a few hundred to a few thousand laser pulses were typically applied to accumulate a mass spectrum. Mass spectra were acquired using TOFMA software (developed by V. Spicer and W. Ens at the University of Manitoba, Winnipeg, CA). Data files were processed with MoverZ software (ver. 2001.02.13; Genomics Solutions, Ann Arbor, MI, USA). Mass resolution of the analysis was about 10,000 (FWHM) and mass accuracy better than $50 \mathrm{ppm}$ when an external calibration was applied using ions of DHB matrix and a small peptide (e.g., bradykinin fragment 1-7). Using internal calibration mass accuracy could be improved to about $10 \mathrm{ppm}$.

Experiments with the QSTAR instrument allowed detection of HCs after adjusting the buffer gas pressure in the ion source to 4-5 mbar of argon and ion extraction conditions. The first adjustment required four modifications to the ion source: (1) replacing the standard ion extraction cone with a central aperture of $4 \mathrm{~mm}$ with one of $1.5 \mathrm{~mm}$ diameter; (2) shutting off a vacuum leak between ion source and quadrupole region (the leaks were intended for standard operation of the oMALDI 2 source); (3) closing a second leak in the curtain gas tube, and (4) adding an additional gas supply line for the Q0 quadrupole region. For HC analysis, "soft" ion extraction conditions were established by setting the "focusing potential" to not more than $20 \mathrm{~V}$, "declustering potential 2 " to values between 5 and $7 \mathrm{~V}$, and the "quadrupole rod offset" to 6-14 V.

Elemental composition assignments of detected ions were made based on the assumptions that theoretical and experimental mass values correspond within $50 \mathrm{ppm}$ (using external calibration) and that ions are comprised of $\mathrm{C}, \mathrm{O}, \mathrm{H}$, $\mathrm{Na}$, and $\mathrm{K}$. For the queen bee, identification of mandibular pheromones was based on previously published literature (see text for references).

\section{UV Absorption Spectroscopy}

Absorption spectroscopy was performed with a 2101-PC spectrometer (Shimadzu, Duisburg, Germany). Whole wings from female $D$. melanogaster were attached to the surface of a UV quartz cuvette.

\section{Results and Discussion}

\section{General Features of the UV-/IR-LDI MS Analysis of Endogenous Compounds}

Endogenously expressed unsaturated aliphatic HCs and oxygen-bearing $\mathrm{HCs}$ can be detected directly from the cuticular surface of individual insects using UV-LDI MS in the absence of any applied chemical matrix. Two typical UV-LDI mass spectra obtained from an adult female and a male D. melanogaster fruit fly, respectively, are shown in Figure 1a and b. Nine hundred laser pulses were applied to one site on the cuticle. Molecular ions of TAGs, FAs, and saccharides are also observed. Species with potassium adducts $[\mathrm{M}+\mathrm{K}]^{+}$formed the base peaks in all mass spectra of $D$. melanogaster recorded in the positive ion mode, presumably because of a high concentration of potassium in the fly cuticle. Additionally, species with sodium adducts $[\mathrm{M}+\mathrm{Na}]^{+}$are detected, albeit with lower signal intensities. Alkanes were not detected under any instrumental settings, likely due to low cation affinities exhibited by fully saturated compounds. Neither HC nor TAG ions were detected in negative ion mode. A comprehensive list of $\mathrm{HC}$ ions detected from the fly cuticle is provided in reference [3].

\section{Influence of the Source Pressure}

The buffer gas pressure in the ion source contributes significantly to the detection of HCs. While intense HC ion signals are recorded at elevated gas pressures above $\sim 1.2$ mbar (e.g., the spectra shown in Figure 1a and b were recorded at 2 mbar of argon), $\mathrm{HC}$ signals are not detected at pressures below 1 mbar (Figure 1c). More polar oxygenated HCs are occasionally detected with signal intensities that are on the order of the chemical background noise. Normally, pressure settings just below $1 \mathrm{mbar}$, along with an extraction voltage in the $50 \mathrm{~V}$ range, provide an optimum for standard MALDI o-TOF MS analysis of compounds like peptides and polar lipids $[9,10]$. The general $\mathrm{HC}$ signal intensitypressure dependence is displayed in Figure 1d, demonstrating a dramatic increase in $\mathrm{HC}$ ion yield above a threshold pressure around 1.2 mbar, before a saturation plateau is reached at $\sim 1.6$ mbar. In addition to argon, nitrogen and methane were also tested as buffer gases and found to provide qualitatively similar results. The data illustrate the importance of establishing sufficient collisional cooling [8, $11,12]$ conditions for the stabilization of labile hydrocarboncation complexes. Notably, signal intensities of triglyceride 

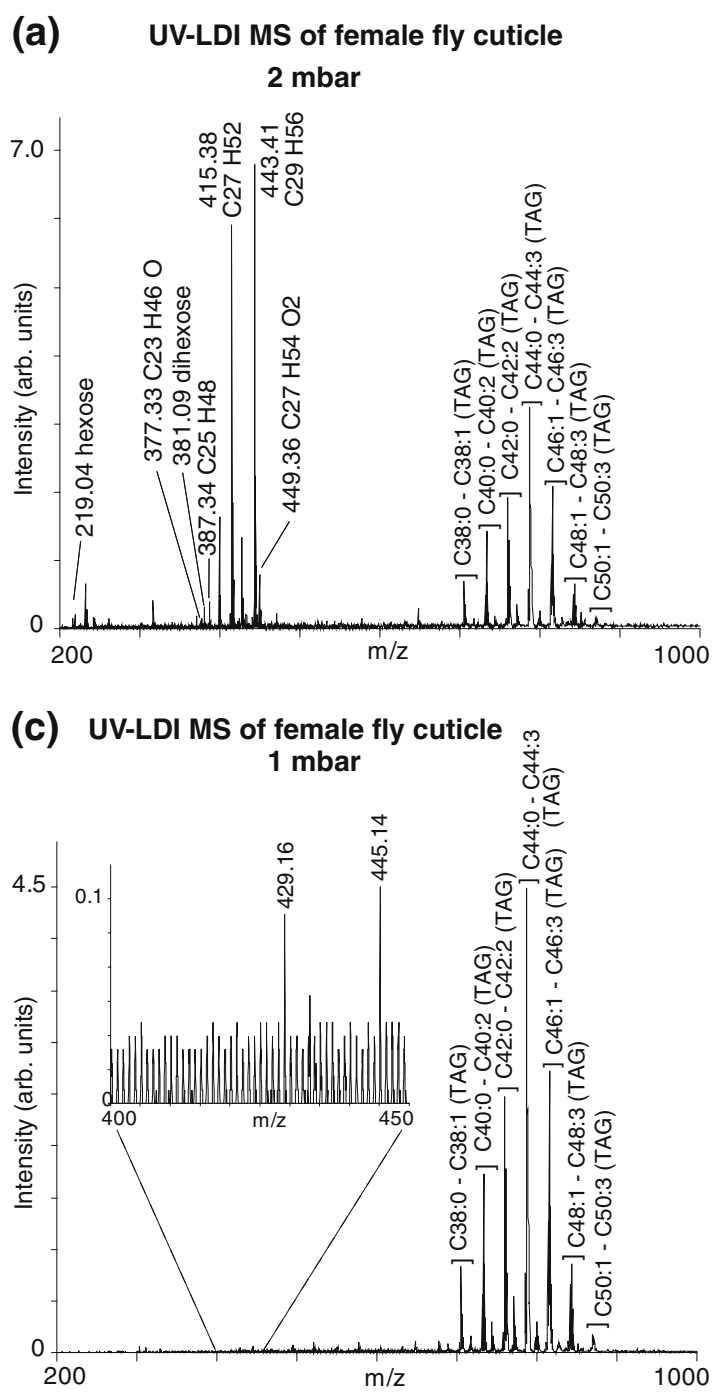

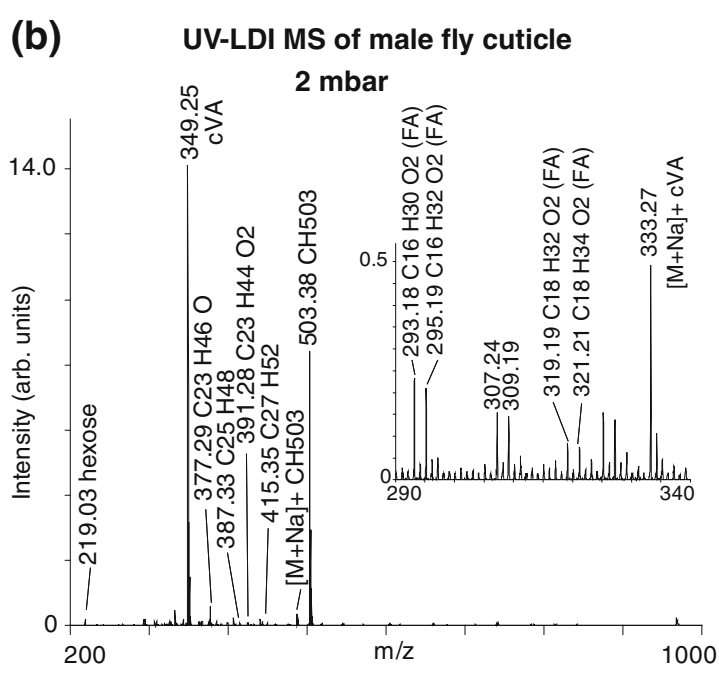

(d) HC signal vs. pressure dependency

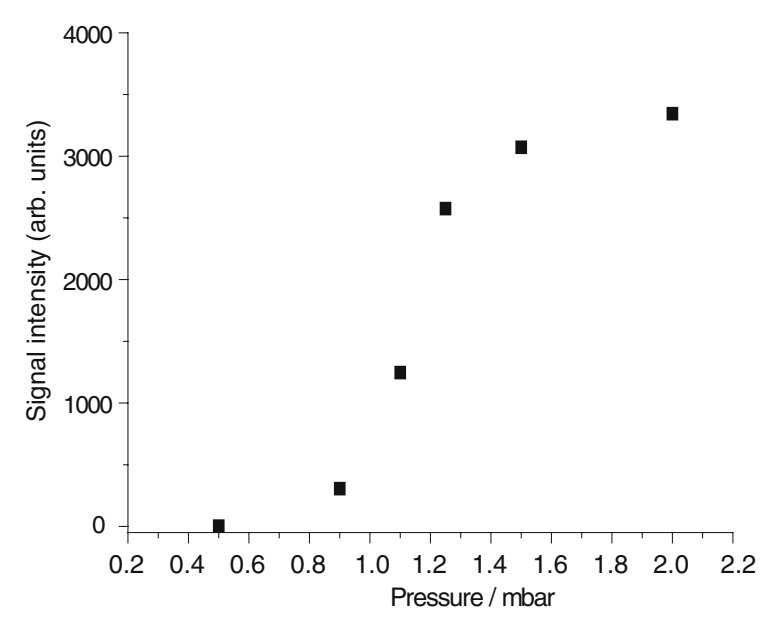

Figure 1. Representative LDI-o-TOF mass spectra acquired from the cuticle of Drosophila melanogaster fruit flies under different experimental conditions. (a) UV-LDI mass spectrum acquired from a virgin female fly at a buffer gas pressure of $2 \mathrm{mbar}$ of argon in the ion source (900 laser shots applied). The major hydrocarbon (HC) and triacylglyceride (TAG) ions are labeled. The total number of $\mathrm{C}$ atoms comprising the three fatty acid residues followed by the overall number of $\mathrm{C}-\mathrm{C}$ double bonds is indicated for each TAG species. The $\mathrm{N}_{2}$ laser $(\lambda=337 \mathrm{~nm})$ was used for UV-LDI MS. (b) UV-LDI mass spectrum acquired from a male fly at a buffer gas pressure of 2 mbar of argon in the ion source. Two known male pheromones (cVA and CH503) and several fatty acids (FA) are detected. Signals corresponding to TAGs are not present in this particular spectrum but would gradually be detected when more laser pulses were applied at a distinct position (see Figure 2). (c) HC ion signals are detected at low intensity after lowering the source pressure to values below 1 mbar. TAG ions are still observed. (d) Dependence of HC ion yield on argon buffer gas pressure was evaluated for two major female compounds, hexacosadiene $\left(\mathrm{C}_{27} \mathrm{H}_{52}\right)$ and nonacosadiene $\left(\mathrm{C}_{29} \mathrm{H}_{56}\right)$

ions are much less affected by the source pressure. These species are detected at pressures below 1 mbar (Figure 1c).

Tests with a commercial o-TOF instrument (QSTAR) allowed successful detection of HCs after adjusting the buffer gas pressure in the ion source to 4-5 mbar or argon and that of some ion extraction conditions (see the Experimental section). Before these modifications were made, only very low and less reproducible $\mathrm{HC}$ ion intensities were obtained for some major HC compounds (data not shown). In the absence of sufficient pressure conditions within the ion source, measurements with other o-TOF and axial instruments from different suppliers also produced only low intensity signals for the most abundant oxygen-containing HCs (data not shown).

\section{Features of IR-LDI MS Versus UV-LDI MS}

Analysis of insect cuticles with an Er:YAG IR laser for direct tissue analysis [13] did not provide advantages for the analysis of $\mathrm{HC}$ compounds (see Supplemental 
Figure S1). As with UV analysis, IR-LDI MS analysis of male flies at 2 mbar of argon produced signals corresponding to two major acetylated sex pheromones, 11cis-vaccenyl acetate (cVA) and 3-O-acetyl-1,3-dihydroxy-octacosa-11,19(Z,Z)-diene (CH503; [14]). TAGs, FAs, and FA methyl esters (FAME) were also detected. Low intensity signals corresponding to oxygen-containing HCs and dienes were observed using both methods though overall signal intensity was higher using the UV laser.

With respect to tissue profiling, one distinct advantage of using a flat-top UV laser beam is that only a few monolayers of material are presumably desorbed with each laser pulse [15]. Therefore, several hundred to thousands of laser pulses can typically be applied at the same position without any visible damage of the cuticle. However, a gradual change in the signal composition, along with a steady decline in intensity of surface HCs, is notable and indicates a gradual removal of surface layers (Figure 2). Sub-surface compounds, in particular TAGs, are increasingly desorbed and ionized as the number of laser shots applied to one spot increases (inset in Figure 2). These data demonstrate that a flat-top UV-beam may be used to record both a molecular surface profile and, by allowing irradiation with a large number of laser shots, a depth profile in a controlled fashion. In contrast to analysis with a UV laser, the IR laser ablates substantially larger amounts of material. The ablation depth per IR laser pulse can be estimated to be on the order of $1-10 \mu \mathrm{m}$ depending on laser fluence [9]. Thus, a more rapid decline in the signal intensities of surface $\mathrm{HC}$ ions is observed with an increasing number of applied IR laser shots (data not shown). In the future, controlled ablation with an IR-laser beam could provide a means for imaging chemical composition beneath the cuticular surface.

\section{Spatial Resolution}

Assuming that ions are emitted solely from irradiated areas, the spatial resolution of the UV-LDI MS analysis is approximately $200 \mu \mathrm{m}$, corresponding to the laser spot diameter [3]. In experiments where the focal laser spot was reduced to a diameter of $100 \mu \mathrm{m}$ by utilizing a second optical fiber with smaller core diameter, no noticeable loss of sensitivity was observed (data not shown). We assume that a spatial resolution in the low $10 \mu \mathrm{m}$ range could be achieved if further modifications were made (e.g., by employing fibers with core diameters in the $10 \mu \mathrm{m}$-range [16]). This could allow spatiallyresolved probing of fine features of the insect body like glands. Automated MS imaging of tissue is not possible in the current o-TOF mass spectrometer system. Nevertheless, profiling by UV-LDI MS is able to detect distinct chemical profiles from different anatomical regions. For example, the anogenital region of male flies shows high local expression of sex pheromones that is distinct from other regions on the fly body [3]. In the future, fine-scale MS imaging experiments may allow us to detect microdomains of specialized $\mathrm{HC}$ expression.

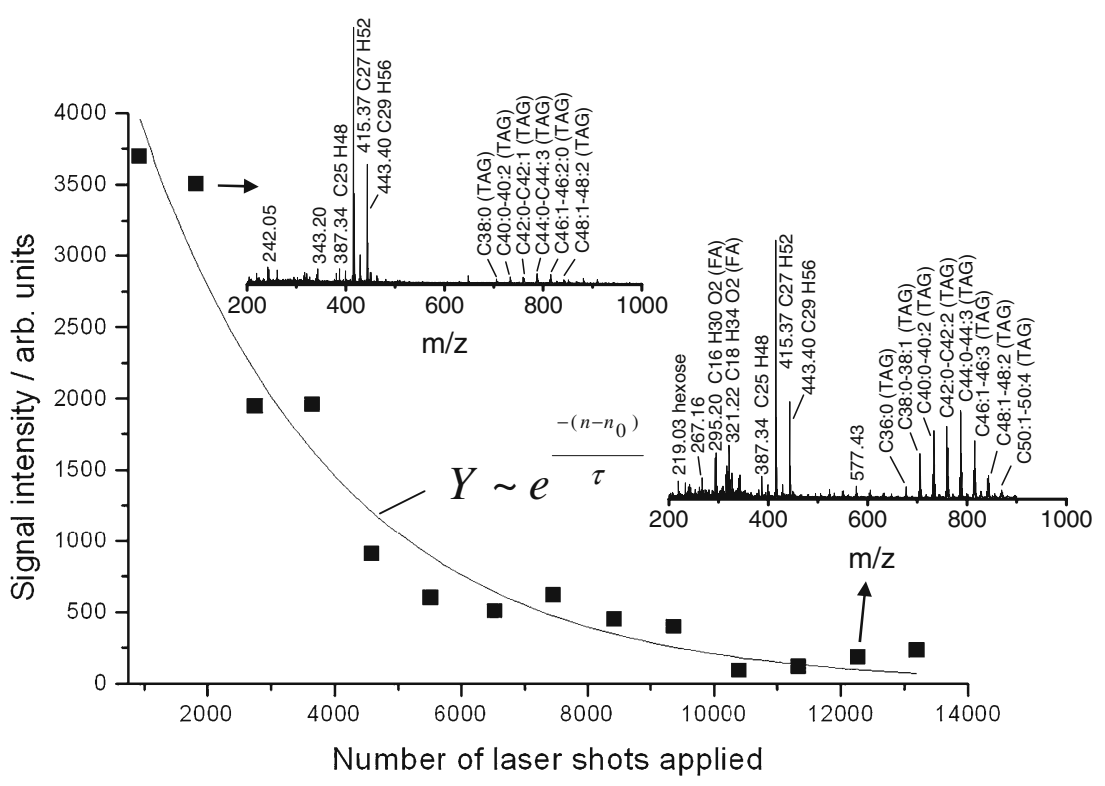

Figure 2. Signal intensities of heptacosadiene $\left(\mathrm{C}_{27} \mathrm{H}_{52}\right)$ and nonacosadiene $\left(\mathrm{C}_{29} \mathrm{H}_{56}\right)$ ions as a function of number of $U V$ laser pulses $(\lambda=337 \mathrm{~nm})$ applied to one spot on the back of a female $D$. melanogaster. The combined signal intensities $[\mathrm{M}+\mathrm{K}]^{+}$of the two $\mathrm{HC}$ compounds detected at $\mathrm{m} / \mathrm{z}$ values of 415.37 and 443.40 , respectively, were averaged over bins of 900 laser pulses (10.5 $\mu \mathrm{J}$ per pulse). The insets show the mass spectra acquired during laser pulses 900-1800 and over the same number of 900 laser pulses after approximately 12000 pre-exposures. The solid line represents a best fit to an empirical formula of the form $Y=\exp \left[-\left(n-n_{0}\right) / \tau\right]$, where $Y$ is the ion yield, $n$ the number of laser pulses, and $\mathrm{n}_{0}$ and $\tau$ are fit parameters. A best fit is obtained for values of $n_{0}=930, \tau=3080$ 


\section{Detection of Endogenous Hydrocarbons and Triglycerides from Other Arthropods}

Direct UV-LDI o-TOF mass spectrometry of other arthropods produced mass spectra that displayed a rich and diverse composition of HCs, FAs, and TAGs. Examples of spectra obtained from the cuticles of various insects from the Hymenoptera, Diptera, and Hemiptera orders are displayed in Figure 3. Other examples of profiles from arthropods are shown in Supplemental Figure S2. As with UV-LDI MS analysis of fruit flies, ions representing long chain $\mathrm{HC}$ species, some of which contain oxygen moieties, are generated from the cuticle of these animals. Signal assignments are made based on exact mass measurements (see Supplemental Tables S1-4). No attempt has been made yet to more fully characterize the chemical profiles. The samples ranged in thickness from approximately $1 \mathrm{~mm}$ (Drosophila) to $10 \mathrm{~mm}$ (queen bees) and in length, from 1-2 $\mathrm{mm}$
(Drosophila) to $20 \mathrm{~mm}$ (spiders, butterfly wings). With the exception of fruit flies, all other organisms required dissection of individual body parts in order to fit within the dimensions of the sample plate. Future refinements such as a deeper indentation in the sample plate, retractable sample plate holder, and adjustable position of the focal laser spot size could allow for chemical imaging of intact larger animals.

Interestingly, some insects yielded sparse mass spectra, displaying only low $\mathrm{HC}$ ion abundances. For example, UV-LDI mass spectra acquired from worker bees displayed only few signals (data not shown). The likely reason for this observation is that the cuticular $\mathrm{HC}$ composition of this species is mostly comprised of alkanes [17], compounds that are not ionized under UV- or IR-LDI conditions. Queen bees express a richer chemical profile near pheromone-producing glands in the (a)

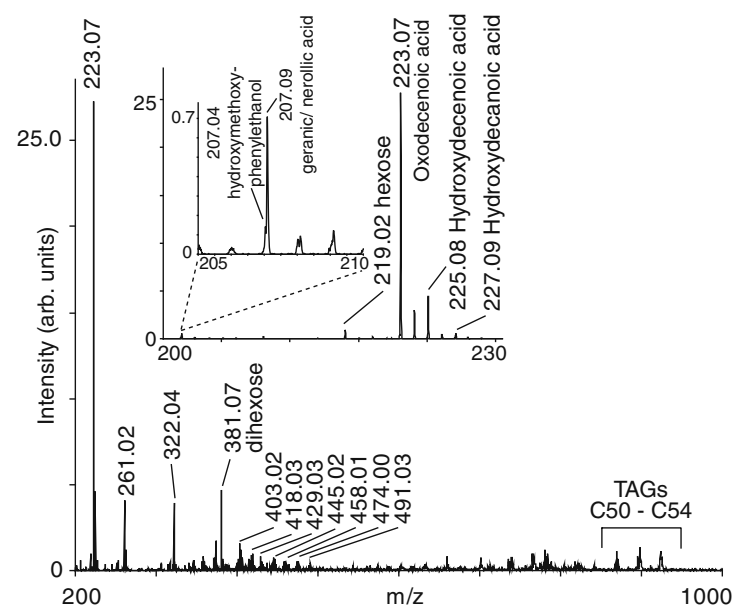

(b)

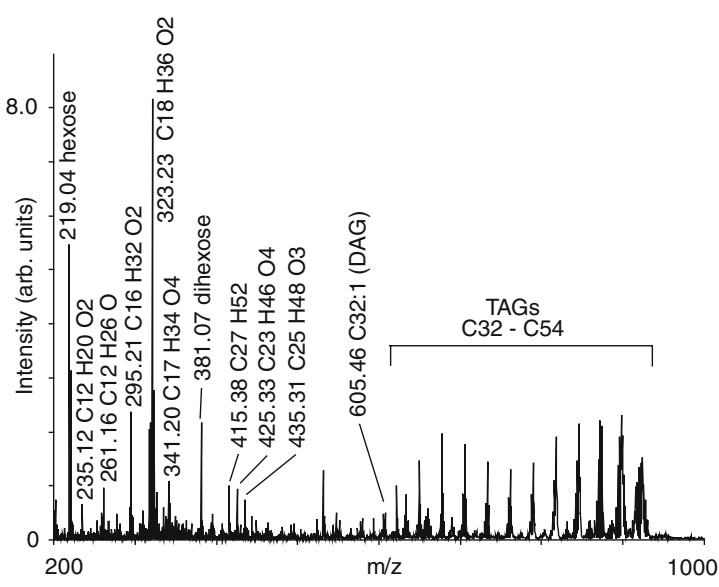

(d)

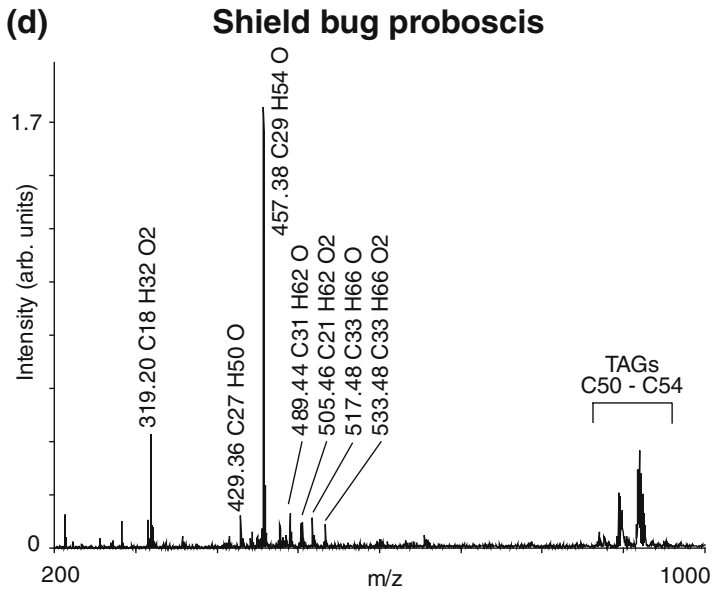

Figure 3. UV-LDI o-TOF mass spectra acquired from the cuticle of various insects from the following orders: (a), (b) Hymenoptera, (c) Diptera, and (d) Hemiptera. Major ion species observed in each spectrum are tentatively assigned. Peak assignments and mass error for each spectrum can be found in Supplemental Tables S3-6. Unless otherwise noted, all signals correspond to molecules bearing a potassium adduct $[\mathrm{M}+\mathrm{K}]^{+}$. Animals were not characterized with respect to their sex and family 
mandible and abdomen [18]. Accordingly, signals corresponding to three fatty acids comprising part of the queen mandibular pheromone (QMP) [18] are detected from the jaw of a queen bee: oxodecenoic acid, hydroxydecenoic acid, hydroxydecanoic acid (Figure 3a; Supplemental Table S1). Small amounts of hydroxydecenoic acid are also found when the head of a worker bee was analyzed (data not shown). Aromatic components hydroxymethoxyphenylethanol (Figure 3a, inset) and methyl-p-hydroxybenzoate (theor. $\mathrm{m} / \mathrm{z} 191.01$; not displayed in Figure 3a), two other major components of the QMP, are detected on the queen bee mandible with low intensity, in addition to geranic and/or isobaric nerolic acid, known pheromones involved in bee aggregation behavior [19]. Other signals (e.g., a series of ions between 400 and $500 \mathrm{Da}$ exhibiting low mass increments) have so far not been identified.

\section{Fly-Assisted Laser Desorption Ionization (FALDI) of Exogenous Compounds}

To investigate whether the fly body itself could be used as a substrate for facilitating desorption/ionization of lipids by "FALDI," synthetic hydrocarbons and crude extract of fly cuticle were applied to individually dissected insect wings and other body parts. Signals corresponding to the following synthetic $\mathrm{HC}$ standards were detected as $[\mathrm{M}+\mathrm{K}]^{+}$species when applied to a fly wing as test compounds (Figure 4a): hexadecanol $\left(\mathrm{C}_{16} \mathrm{H}_{32} \mathrm{O}\right.$, theor. $m / z$ of the $[\mathrm{M}+\mathrm{K}]^{+}$ion species 279.21), hexadecenal $\left(\mathrm{C}_{16} \mathrm{H}_{30} \mathrm{O}\right.$, theor. $m / z$ 277.19), and hexadecenyl acetate $\left(\mathrm{C}_{18} \mathrm{H}_{34} \mathrm{O}_{2}\right.$, theor. $\left.\mathrm{m} / \mathrm{z} 321.22\right)$. Fly wings provide flat surfaces and contain only low concentrations of TAGs and, thus, serve as suitable substrates for FALDI MS analysis. However, other parts of the fly (like legs) were also tested and each was found to be capable of generating ions when used as substrate. Endogenous hydrocarbons can be removed by washing flies briefly in hexane, thereby simplifying the analysis of complex HC mixtures. One disadvantage of the washing step is that it makes the fly wings more fragile. In the case of Drosophila, we eventually also observed an increase of some new background HC signals, likely derived from subcutaneous layers. Despite these drawbacks, amounts of at least tens of pmol of the above HC compounds could still be detected using hexanewashed fly wings.

Figure $4 \mathrm{~b}$ shows that crude cuticular extract from Drosophila can also be analyzed when spotted onto wings of other insects. All major classes of lipids detected by UVLDI MS endogenously on Drosophila (mono- and disaturated hydrocarbons, oxygen-containing $\mathrm{HCs}$, and triglycerides) were observed after application of crude fly extract on a wasp wing. In addition to providing a larger surface area, bee and wasp wings are particularly advantageous for facilitating FALDI MS analysis since these parts generally produce little endogenous signal and do not require washing.
As with detection of endogenous compounds by direct UVLDI MS, successful FALDI MS analysis of HCs requires elevated pressure settings.

\section{Comparison with MALDI MS}

When cuticular extract from the equivalent of 1 fly was analyzed using DHB as a MALDI matrix, $[\mathrm{M}+\mathrm{K}]^{+}$signals of two of the most abundant oxygen-containing HCs could be detected but with at least 10 -fold lower intensity compared to analysis with an insect wing (data not shown). Monoenes and dienes without oxygen atoms were not detected. When the HC standards were analyzed by MALDI MS, compounds were detected as sodium adduct ions, albeit with intensities that were at least a factor of 10 lower than those of the $[\mathrm{M}+\mathrm{K}]^{+}$signals recorded using fly wings (data not shown).

MALDI MS analysis of cuticular HCs from extract has previously been demonstrated with lithiated dihydroxybenzoate matrix using a standard "axial"-MALDI-TOF mass spectrometer operated with high vacuum in the ion source [20]. Notably, this approach allowed the analysis of saturated HCs, detected in form of lithium adducts, albeit with sensitivity limits that appear to fall into the low $\mu \mathrm{g}$ range [21]. Using the same matrix for "MALDI imaging" of Drosophila wings the two major dienes, hexacosadiene and nonacosadiene, found in female fly cuticle appear to be detected just above noise level [22]. Other laser-based approaches utilize adduct formation of $\mathrm{HC}$ molecules with silver ions [23, 24]. However, the occurrence of two natural silver isotopes at 107 and $109 \mathrm{u}$ and their tendency to form oligomers upon laser desorption can produce rather complex mass spectra and obscure resolution of HCs differing by two hydrogen atoms.

\section{FALDI MS of Fatty Acids, Fatty Acid Methyl Esters, and Triglycerides}

We tested whether FALDI MS is suitable for the analysis of FAs, FAMEs, and TAGs and examined the dependence of signal intensities on buffer gas pressure values. Signals corresponding to ions from synthetic octadecenoic acid and octadecenoic acid methyl ester were detected using insect wing as a substrate down to amounts in the low $10 \mathrm{pmol}$ range (Supplemental Figure S3). The limit of detection is approximately 10 pmol for the FA and FAME standards (Supplemental Figure S3, inset). At $0.6 \mathrm{mbar}$, signal intensities were 6-8 times lower (data not shown). Mass spectra acquired from MALDI preparations with DHB matrix showed signals for these two compounds as well, though the sensitivity limit was at 200 pmol in this case (data not shown). TAGs can also be detected using FALDI. A drop of commercial olive oil applied to a bee wing produced a spectrum dominated by a distribution of triglycerides, the most abundant constituents in the oil (Figure 4c). 
(a) Fly wing $+1-3 \mathrm{nmol} \mathrm{HC}$ standards
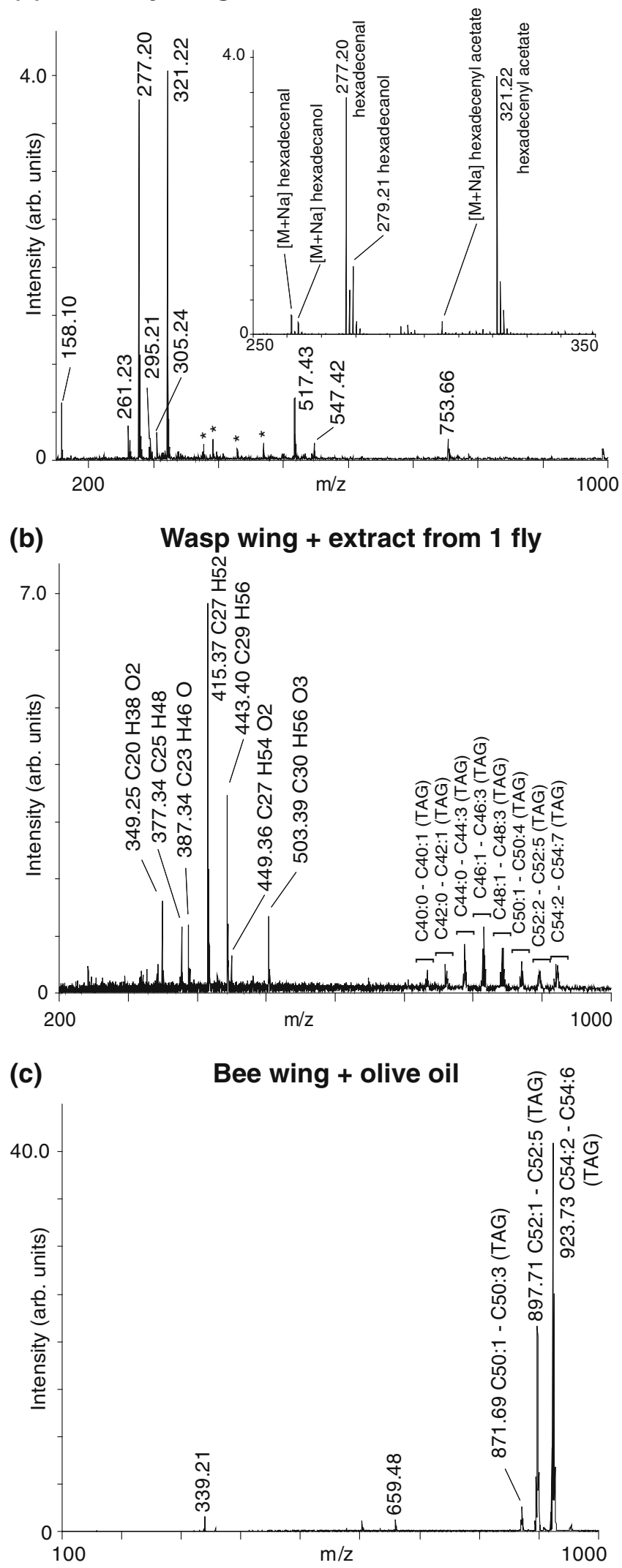

Figure 4. Examples of FALDI mass spectra of lipids. (a) 1$3 \mathrm{nmol}$ each of hexadecanol $\left(\mathrm{C}_{16} \mathrm{H}_{32} \mathrm{O}\right.$, theor. $\mathrm{m} / \mathrm{z}$ of the monoisotopic $[\mathrm{M}+\mathrm{K}]^{+}$ion species: 279.21), hexadecenal $\left(\mathrm{C}_{16} \mathrm{H}_{30} \mathrm{O}\right.$, theor. $\left.\mathrm{m} / \mathrm{z} 277.19\right)$, and hexadecenyl acetate $\left(\mathrm{C}_{18} \mathrm{H}_{34} \mathrm{O}_{2}\right.$, theor. $\left.\mathrm{m} / \mathrm{z} 321.22\right)$ applied to an untreated fly wing. (b) Crude cuticular extract from the equivalent of 1 female Drosophila melanogaster spotted onto an untreated wasp wing. (c) A drop of olive oil applied to a bee wing. All mass spectra were recorded using the $\mathrm{N}_{2}$ laser $(\lambda=337 \mathrm{~nm})$ and a buffer gas pressure of $\sim 2$ mbar of argon. Unless otherwise noted, all signals correspond to molecules with a potassium adduct $[\mathrm{M}+\mathrm{K}]^{+}$; * denotes endogenous ion species from the wing

\section{FALDI MS of Peptides}

Small peptides (approximately up to $1 \mu$ mol were applied to wings) were also tested as analytes. Approximately up to $1 \mu \mathrm{mol}$ were applied to wings and did not produce any detectable signal at any pressure setting. It is likely that there is a different interaction of these hydrophilic analytes with the hydrophobic surface and different ionization pathways. While lipids are detected as alkali-adducts, peptides are most typically detected by MALDI MS as protonated species $[\mathrm{M}+\mathrm{H}]^{+}$. The lacking proton donor in the matrix-less LDI preparation may account for the failure to analyze peptides by FALDI MS. However, further factors must be involved since also $[\mathrm{M}+\mathrm{Na}]^{+}$or $[\mathrm{M}+\mathrm{K}]^{+}$signals of the peptides are not detected.

\section{Possible Use of FALDI MS as an Analytical Method}

Chemical matrices such as DHB and 4- $\alpha$-cyano-hydroxycinnamic acid (CHCA) are commonly used for the analysis of polar lipids [25] and other biomolecules by MALDI MS. While small molecules and, in particular, metabolites can be analyzed by MALDI MS [26, 27], the presence of matrix-associated signals in the lower mass region of the spectra can heavily interfere with analyte-derived signals of small molecules. To circumvent this problem, a number of "matrix-free" preparation protocols have been developed. For example, etched silica surfaces for desorption/ionization on silicon (DIOS [28]) and nanowire-assisted LDI (NALDI [29]) have been introduced, to name only a few. These materials share a strong optical absorption at the excitation laser wavelength and provide a large specific surface area. However, these substrates are relatively costly and require care regarding proper storage and handling in order to prevent contamination by adsorbing compounds. Insect wings could provide a biomimetic model for the production of synthetic materials exhibiting the analytical features seen in these initial experiments.

Signal Intensities of Endogenous Hydrocarbon Ions as a Function of Laser Wavelength and Pulse Energy

To better understand the physicochemical mechanisms underlying the UV-LDI MS analysis of fly cuticle, we 
studied the contribution of laser wavelength and optical properties of the cuticle. The dorsal surfaces of female flies were irradiated and the excitation laser wavelength varied between 280 and $380 \mathrm{~nm}$. "Threshold" pulse energies necessary to provoke an overall ion count/se of $1000 \pm 300$ were recorded as a function of wavelength in order to identify whether a specific endogenously-expressed chromophore may facilitate deposition of the laser pulse energy and desorption of cuticular compounds. If this were the case, enhanced signal intensities are likely to be observed within a limited wavelength range, corresponding to the peak absorption of the chromophore. Hydrocarbon and triglycerides are nonabsorbing, i.e., transparent throughout the probed laser wavelength range.

The data displayed in Figure 5a reveal a relatively weak dependence on excitation laser wavelength. The pulse energy required to reach an ion count of $1000 \pm 300$ varied between 4 and $6 \mu \mathrm{J}$ and mass spectra recorded at individual wavelengths look essentially identical (insets in Figure 5a). These pulse energies on the sample surface translate to fluences (pulse energy per area) of $110-170 \mathrm{~J} / \mathrm{m}^{2}$ if a laser spot size of $200 \times 230 \mu \mathrm{m}^{2}$ is used for the calculation. These fluence values are comparable to those employed in typical MALDI experiments. For example, the threshold fluence for ion detection with a DHB matrix has been determined to be about $100 \mathrm{~J} / \mathrm{m}^{2}$ [7], while that of CHCA is about a factor of 2-3 times lower [30]. Because of the curved shape of the fly body and undefined height of the sample position, we note that the spot size on the cuticle is estimated with a relatively large error; therefore, we prefer to use pulse energy values throughout this manuscript instead of fluences.

A few wavelength-dependent fine features were notable. For example, for wavelengths below $300 \mathrm{~nm}$, the cuticle appears to be disrupted by fewer applied laser pulses, giving rise to an earlier onset of the detection of subsurface TAGs. This observation might be attributed to an enhanced photochemistry at high photon energies. The best results in terms of the reproducibility, ease of spectrum generation, and minimal destruction to the cuticles were produced with wavelengths of $340 \mathrm{~nm}$ and higher. Despite similar "threshold" pulse energies of approximately $4-5 \mu \mathrm{J}$ at $350-380 \mathrm{~nm}$ and $280-300 \mathrm{~nm}$, no detectable damage to fly cuticles was observed for the longer wavelengths.

In one experiment, we also tested a fundamental wavelength of the dye laser at $560 \mathrm{~nm}$. Desorption/ionization of $\mathrm{HC}$ and TAG ions was possible with this visible radiation but required a threshold pulse energy of $\sim 66 \mu \mathrm{J}$, about a factor of 10 higher than in the probed UV region. The elevated threshold is likely to be attributed to a reduced absorption of laser light by the cuticle.

\section{Optical Properties of Fly Cuticle}

Iridescent coloring of insect cuticle and wings is a wellstudied phenomenon not only because of its intrinsic aesthetic appeal but also because of applications to material
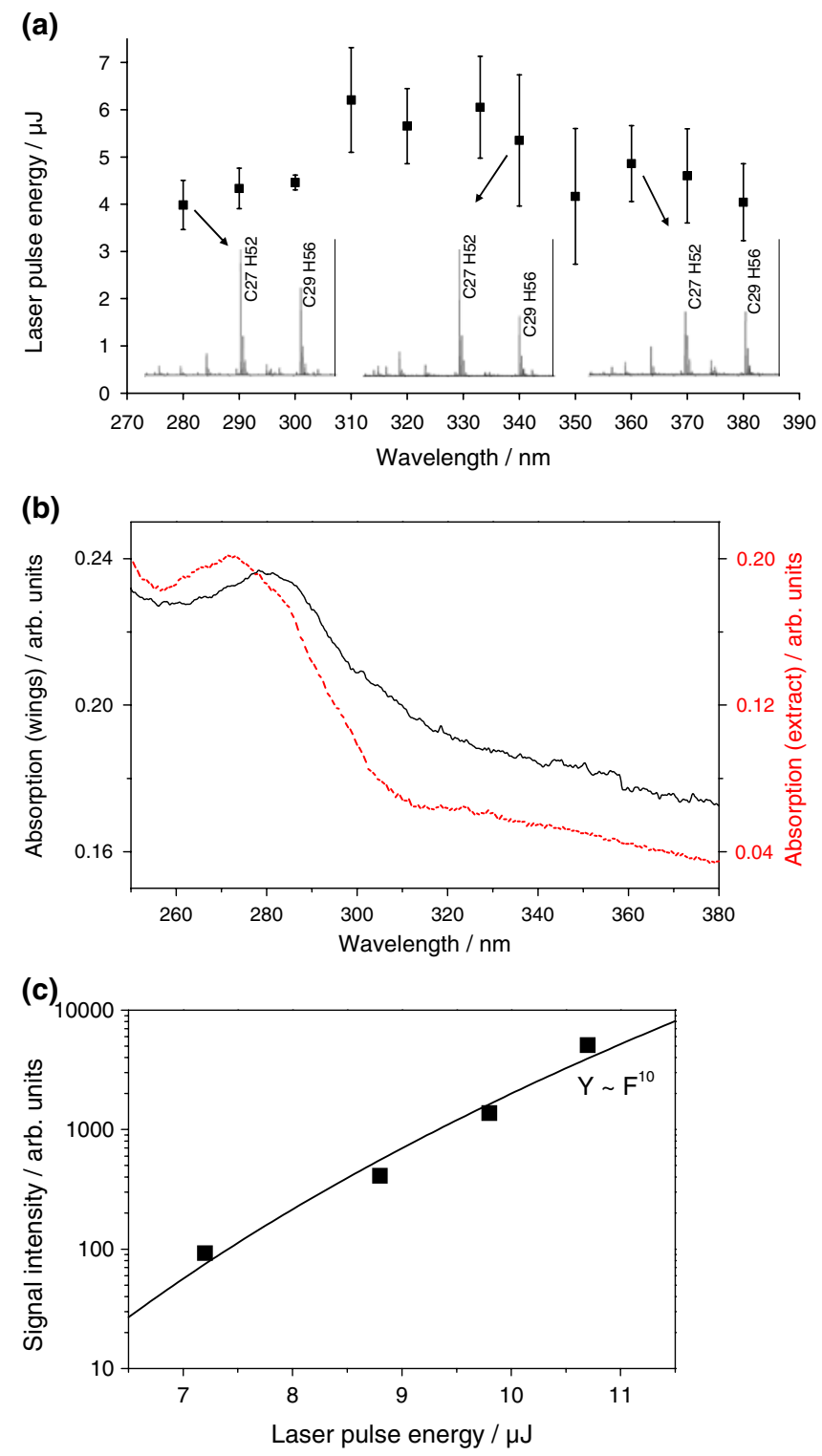

Figure 5. (a) Wavelength-dependent pulse energies for the generation of ion counts of $\sim 1000( \pm 300)$; ion counts refer to all ions detected regardless of their identity. lons were generated by irradiating the dorsal surface (back) of female D. melanogaster $(n=5)$ with the dye laser beam (900 exposures per data point, $10.5 \mu \mathrm{J}$ per pulse). Representative mass spectra obtained at wavelengths of 280,340 , and $360 \mathrm{~nm}$, respectively, are displayed in the insets. (b) UV absorbance spectrum of whole $D$. melanogaster wings (black solid line) and cuticular extract (red dashed red line). (c) Yield of hydrocarbon ions as a function of laser pulse energy. Data were recorded from the dorsal surface (back) of female $D$. melanogaster flies using an $\mathrm{N}_{2}$ laser $(\lambda=337 \mathrm{~nm})$; 900 laser shots were applied per data point and the combined signal intensities of potassium-bearing heptacosadiene $\left(\mathrm{C}_{27} \mathrm{H}_{52}\right)$ and nonacosadiene $\left(\mathrm{C}_{29} \mathrm{H}_{56}\right)$ detected at $\mathrm{m} / \mathrm{z} 415.37$ and 443.40 , respectively, were evaluated. Pulse energies refer to those on the sample. The solid line represents a fit to an empirical function of the form $Y=E_{p}^{m}$, where $Y$ is the ion yield, $E_{p}$ the laser pulse energy, and $\mathrm{m}$ a fit parameter 
science and detection technology that may arise from understanding the underlying optical processes. Insect cuticle consists of lipids, phenolic compounds, and chitin filaments that are embedded in a protein matrix [31-33]. Depending on the species, coloring can be caused by chromophores and/or by "structural coloring" imparted from microstructures [34]. For example, pigmentation in flies, bees, and wasps is attributed to the presence of aromatic compounds heavily involved in cross-linking of the extracellular matrix, as well as to the occurrence of melanin in black-colored cuticle [35]. In the case of moth and butterfly wings, electron microscopy has revealed that cuticular structures are arrayed in multilayers or contain photonic crystals [36], both of which produce optical interference and can contribute to iridescent coloring.

To begin to investigate the optical properties of fly cuticle, we compared the light transmission properties of dissected fly wings to those of crude cuticle extract. An absorption spectrum recorded from whole Drosophila wings in transmission mode is displayed in Figure $5 \mathrm{~b}$. The data reveal a local maximum in the optical absorption at approximately $280 \mathrm{~nm}$, likely corresponding to the peak absorption of small aromatic molecules like phenols in the cuticle and to aromatic tryptophan/tyrosine residues in proteins. The data were not corrected for scattering which, in addition to absorption by melanin, is likely to partially account for the monotonous increase in absorption with decreasing wavelength. One should note, however, that the optical properties of uncolored wings may only partially reflect those of the pigmented black and tan-colored cuticle. Cuticular chloroform/methanol extract of whole Drosophila displayed a blue-shift of the peak absorption to $272 \mathrm{~nm}$ (dashed line in Figure 5b). The different absorption curves of fly wing and chloroform/methanol extract indicate that only some chromophores were extracted. In particular, compounds cross-linked to proteins are unlikely to be dissolved. The possibility that cross-linked compounds could contribute to the optical properties of cuticle is supported by observations that wings that are sonicated for $30 \mathrm{~min}$ in methanol, placed in boiling water for $5 \mathrm{~min}$, or microwaved for several minutes still retain FALDI properties (data not shown). Comparison of spectroscopic data with the wavelength dependence of the MS threshold pulse energies shows some correlation for the short wavelength side in that the increased optical absorption below $\sim 300 \mathrm{~nm}$ (Figure 5a) appears to be reflected by a decreased energy required to reach the ion count threshold. However, the fact that almost identical threshold pulse energies are observed between 350 and $380 \mathrm{~nm}$ is not reflected in the spectroscopic data. Evidence for the contribution of structural coloring, which presumably would lead to more pronounced absorptionwavelength modulations, is not found in the UV or in the visible wavelength range (data not shown).

Future experiments will seek to identify the molecules found on the cuticle that serve as a matrix. In addition, it will be interesting to characterize the wavelength-dependent MS properties of insect wings that exhibit notable structural coloring (e.g., butterfly wings) and to examine whether structural properties may also enhance MS-related attributes.

\section{Ion Signal Intensity as a Function of Laser Pulse Energy}

A second parameter that could reveal fine features underlying FALDI processes is the dependence of HC signal intensity on laser pulse energy. To examine this property, the dorsal cuticles of female flies were irradiated using a $\mathrm{N}_{2}$ laser $(\lambda=337 \mathrm{~nm})$ at various pulse energies above ion generation threshold and the intensity of signals corresponding to two prominent female $\mathrm{HC}$ compounds, hexacosadiene and nonacosadiene, were evaluated (Figure $5 \mathrm{c}$ ). HC signals display a strong exponential increase with laser pulse energy that can be fit to an empirical function of the form $Y=E_{p}^{m}$, where $Y$ is the ion yield, $E_{p}$ the laser pulse energy, and $\mathrm{m}$ a fit parameter. A best fit is obtained with $\mathrm{m} \sim 10$. Notably, essentially the same ion yield-pulse energy dependencies are found in UV-MALDI experiments. Data analysis, including desorbed neutral molecules, showed that this dependence can be attributed to a quasi-thermal evaporation of molecules from near-surface elements convoluted with a gas phase ionization process $[7,15]$.

\section{Desorption Ionization Mechanisms}

Taking the different experimental findings together, we hypothesize that thermal processes are likely to dominate the generation of ions of endogenous and exogenous lipid compounds from insect cuticle by pulsed UV laser desorption/ionization, facilitated by absorption of laser energy by endogenously expressed chromophores. Collisions of neutral HCs and alkali cations in the expanding material plume probably form the basis for the necessary ionization step [37]. These labile cation-bound complexes are stabilized by rapid collisional cooling in the elevated pressure ion source.

\section{Comparison to GC, ESI, and DART MS}

Compared with GC/MS, the standard method for the analysis of cuticular HCs, UV-LDI MS analysis extends the accessible mass range and allows detection of long-chain and more polar HCs such as of hydroxylated and acetylated HCs [3]. Due to the only ns-long excitation laser pulse, gaseous molecules are desorbed as intact cationized molecular ions. It is likely that these compounds would fragment upon evaporation under the quasi-continuous heating conditions found in a GC oven. In contrast to GC/MS, saturated HCs cannot currently be detected by UV-LDI MS. Ultimately, a combination of GC/MS and UV-LDI MS analyses would provide a more comprehensive picture of the cuticular $\mathrm{HC}$ profile of insects and could enable new insights into chemical communication and behavior. 
Many of the compounds that are detected by direct UVLDI mass spectrometry of fly cuticle can also be analyzed from solution using electrospray ionization (ESI) mass spectrometry and by direct desorption in real time (DART) MS [3, 38]. One advantage of these methods is that because protonated molecules can be produced, collisional induced dissociation (CID) can more readily be employed for structural elucidation and detection of functional groups like hydroxyls and acetates in oxygen-containing HCs. In addition, emission of ionic cuticular compounds was recently also observed from live flies when exposed at atmospheric pressure (AP) to a strong electrical field applied between the insects and the inlet cone of an ESI/AP-MALDI mass spectrometer [39]. Future work will address the possibility of whether UV-LDI at AP conditions can be used for the analysis of live flies.

\section{Acknowledgments}

The authors gratefully acknowledge Alexandre Loboda for excellent technical support, Clemens Pöppelmann and Helge Karch for providing worker and queen bee samples, Malcolm Clench and Gottfried Pohlentz for helpful discussions, Edward Kravitz, Franz Hillenkamp, Stefan Berkenkamp, and Sequenom GmbH for support of the project. J.Y.Y. acknowledges a research fellowship by the Alexander von Humboldt foundation and a short-term fellowship by the Human Science Frontier Program Organization. The authors further acknowledge support for this work by the fund Innovative Medical Research of the University of Münster Medical School (grants DR520805 and DR511003), the Singapore National Research Foundation, and the Deutsche Forschungsgemeinschaft (grant DR 416/8-1).

\section{References}

1. Howard, R. W.; Blomquist, G. J. Ecological, behavioral, and biochemical aspects of insect hydrocarbons. Annu. Rev. Entomol. 2005, 50, 371-393.

2. Cook, S. M.; Khan, Z. R.; Pickett, J. A. The use of push-pull strategies in integrated pest management. Annu. Rev. Entomol. 2007, 52, 375400 .

3. Yew, J. Y.; Dreisewerd, K.; Luftmann, H.; Müthing, H.; Pohlentz, G.; Kravitz, E. A. A new male sex pheromone and novel cuticular cues for chemical communication in Drosophila. Curr. Biol. 2009, 19, 1245 1254.

4. de la Paz Fernández, M.; Chan, Y.-B.; Yew, J. Y.; Billeter, J.-C.; Dreisewerd, K.; Levine, J.; Kravitz, E. A. Pheromonal and Behavioral Cues Trigger Male-to-Female Aggression in Drosophila. PLoS Biol. 2010, 8, e1000541.

5. Yew, J. Y.; Dreisewerd, K.; de Olivera, C. C.; Etges, W. J. Malespecific transfer and fine scale spatial differences of newly identified cuticular hydrocarbons and triglycerides in a Drosophila species pair. PLoS ONE 2011, 6, e16898.

6. Soltwisch, J.; Souady, J.; Berkenkamp, S.; Dreisewerd, K. Effect of gas pressure and gas type on the fragmentation of peptide and oligosaccharide ions generated in an elevated pressure UV/IR-MALDI ion source coupled to an orthogonal time-of-flight mass spectrometer. Anal. Chem. 2009, 81, 2921--2934.

7. Dreisewerd, K.; Schürenberg, M.; Karas, M.; Bahr, U.; Hillenkamp, F. Influence of the laser intensity and spot size on the desorption of molecules and ions in matrix-assisted laser desorption ionization with a uniform beam profile. Int. J. Mass Spectrom. Ion Processes 1995, 141, $127-148$.
8. Loboda, A. V.; Ackloo, S.; Chernushevich, I. V. A high-performance matrix-assisted laser desorption/ionization orthogonal time-of-flight mass spectrometer with collisional cooling. Rapid Commun. Mass Spectrom. 2003, 17, 2508-2516.

9. Soltwisch, J.; Dreisewerd, K. Discrimination of isobaric leucine and isoleucine residues and analysis of posttranslational modifications in peptides by MALDI in-source decay mass spectrometry combined with collisional cooling. Anal. Chem. 2010, 82, 5628-5635.

10. Dreisewerd, K.; Lemaire, R.; Pohlentz, G.; Salzet, M.; Wisztorski, M.; Berkenkamp, S.; Fournier, I. Molecular profiling of native and matrixcoated tissue slices from rat brain by infrared and ultraviolet laser desorption/ionization orthogonal time-of-flight mass spectrometry. Anal. Chem. 2007, 79, 2462-2471.

11. Krutchinsky, A. N.; Loboda, A. V.; Spicer, V., L.; Dworschak, R.; Ens, W.; Standing, K. G. Orthogonal injection of matrix-assisted laser desorption/ionization ions into a time-of-flight spectrometer through a collisional damping interface. Rapid Commun. Mass Spectrom. 1998, 12, 508-518.

12. O'Connor, P. B.; Costello, C. E. A high pressure matrix-assisted laser desorption/ionization Fourier transform mass spectrometry ion source for thermal stabilization of labile biomolecules. Rapid Commun. Mass Spectrom. 2001, 15, 1862-1868.

13. Ibáñez, A. J.; Scharte, J.; Bones, P.; Pirkl, A.; Meldau, S.; Baldwin, I. T.; Hillenkamp, F.; Weis, E.; Dreisewerd, K. Rapid metabolic profiling of Nicotiana tabacum defense responses against Phytophthora nicotianae using direct infrared laser desorption ionization mass spectrometry and principal component analysis. Plant Methods 2010, 6, 14.

14. Mori, K.; Shikichi, Y.; Shankar, S.; Yew, J. Y. Pheromone synthesis. Part 244: Synthesis of the racemate and enantiomers of (11Z,19Z)CH503 (3-acetoxy-11,19-octacosadien-1-ol), a new sex pheromone of male Drosophila melanogaster to show its (S)-isomer and racemate as bioactive. Tetrahedron 2010, 66, 7161-7168.

15. Dreisewerd, K. The desorption process in MALDI. Chem. Rev., 2003, 103, 395-426.

16. Qiao, H.; Spicer, V.; Ens, W. The effect of laser profile, fluence, and spot size on sensitivity in orthogonal-injection matrix-assisted laser desorption/ionization time-of-flight mass spectrometry. Rapid Commun. Mass Spectrom. 2008, 22, 2779-2790.

17. Mar, T.; Brill, J.; Bertsch, W.; Fletcher, D. J. C.; Crewe, R. Investigation of cuticular hydrocarbons from selected honeybee populations by gas chromatography with pattern recognition. $J$. Chromat. A 1987, 399, 277-290.

18. Slessor, K. N.; Kaminski, L.-A.; King, G. G. S.: Borden, J. H.; Winston, M. L. Semiochemical basis of the retinue response to queen honey bees. Nature 1988, 332, 354-356.

19. Boch, R.; Shearer, D. A. Identification of nerolic and geranic acids in the Nassanoff pheromone of the honey bee. Nature 1964, 202, 320321.

20. Cvačka, J.; Jiros, P.; Sobotnik, J.; Hanus, R.; Svatoš, A.. Analysis of Insect Cuticular Hydrocarbons using Matrix-Assisted Laser Desorption/ Ionization Mass Spectrometry. J. Chem. Ecol. 2006, 32, 409-434.

21. Cvačka, J.; Svatoš, A. Matrix-assisted laser desorption/ionization analysis of lipids and high molecular weight hydrocarbons with lithium 2,5-dihydroxybenzoate matrix. Rapid Commun. Mass Spectrom. 2003, 17, 2203-2207.

22. Vrkoslav, V.; Muck, A.; Cvačka, J.; Svatoš, A. MALDI imaging of neutral cuticular lipids in insects and plants. J. Am. Soc. Mass Spectrom. 2010, 21, 220-231.

23. Kühn, G.; Weidner, S.; Just, U.; Hohner, G. Characterization of technical waxes: Comparison of chromatographic techniques and matrix-assisted laser-desorption/ionization mass spectrometry. J. Chromatogr. A 1996, 732, 111-117.

24. Dutta, T. K.; Harayama, S. Time-of-flight mass spectrometric analysis of high-molecular-weight alkanes in crude oil by silver nitrate chemical ionization after laser desorption. Anal. Chem. 2001, 73, 864-869.

25. Schiller, J.; Süss, R.; Fuchs, B.; Müller, M.; Zschörnig, O.; Arnold, K. MALDI-TOF MS in lipidomics. Front. Biosci. 2007, 12, 2568-2579.

26. Cohen, L. H.; Gusev, A. I. Small molecule analysis by MALDI mass spectrometry. Anal. Bioanal. Chem. 2002, 373, 571-586.

27. Burrell, M. M.; Earnshaw, C. J.; Clench, M. R. Imaging Matrix Assisted Laser Desorption Ionization Mass Spectrometry: A Technique to Map Plant Metabolites Within Tissues at High Spatial Resolution. J. Exp. Bot. 2007, 58, 757-763. 
28. Lewis, W. G.; Shen, Z.; Finn, M. G.; Siuzdak, G. Desorption/ionization on silicon (DIOS) mass spectrometry: Background and applications. Int J. Mass Spectrom. 2003, 226, 107-116.

29. Kang, M. J.; Pyun, J. C.; Lee, J. C.; Choi, Y. J.: Park, J. H.; Park, J. G.; Lee, J. G.; Choi, H. J. Nanowire-assisted laser desorption and ionization mass spectrometry for quantitative analysis of small molecules. Rapid Commun. Mass Spectrom. 2005, 19, 3166-3170.

30. Westmacott, G.; Ens, W.; Hillenkamp, F.; Dreisewerd, K,; Schürenberg, $M$. The influence of laser fluence on ion yield in matrix-assisted laser desorption ionization mass spectrometry. Int. J. Mass Spectrom. 2002, 221, 67-81.

31. Andersen, S. O.; Peter, M. G.; Roepstorff, P. Cuticular sclerotization in insects. Comp. Biochem. Physiol. B Biochem. Mol. Biol. 1996, 113, 689-705.

32. Hopkins, T. L.; Kramer, K. J. Insect cuticle sclerotization. Ann. Rev Entomol. 1992, 37, 273-302

33. Andersen, S. O. Cuticular sclerotization and tanning. In: Comprehensive Molecular Insect Science Vol. IV. Elsevier Pergamon Press: New York 2005; pp. 145-170.
34. Kinoshita, S.; Yoshioka, S. Structural colors in nature: The role of regularity and irregularity in the structure. Chem. Phys. Chem. 2005, 6, $1442-1459$.

35. Andersen, S. O. Insect cuticular sclerotization: A review. Insect Biochem. Mol. Biol. 2010, 40, 166-178.

36. Saranathan, V.; Osuji, C. O.; Mochrie, S. G. J.; Noh, H.; Narayanan, S.; Sandy, A.; Dufresne, E. R.; Prum, R. O. Structure, Function, and SelfAssembly of Single Network Gyroid (I4132) Photonic Crystals in Butterfly Wing Scales. Proc. Nat. Acad. Sci. U.S.A. 2010, 107, 11676-11681.

37. Wang, B. H.; Dreisewerd, K.; Bahr, U.; Karas, M.; Hillenkamp, F. Gasphase cationization and protonation of neutrals generated by matrixassisted laser desorption. J. Am. Soc. Mass Spectrom. 1993, 4, 393-398.

38. Yew, J. Y.; Cody, R. B.; Kravitz, E. A. Cuticular hydrocarbon analysis of an awake behaving fly using direct analysis in real-time time-of-flight mass spectrometry. Proc. Nat. Acad. Sci. U.S.A. 2008, 105, 7135-7140.

39. Pirkl., A.; Dreisewerd, K.; Yew, J. Y.; König, S. Field-based ion generation from microscale emitters on natural and artificial objects for atmospheric pressure mass spectrometry. Anal. Bioanal. Chem. 2010, 397, 3311-3316 